\title{
Single-Particle Cryo-EM Structure of a Membrane Protein in a Membrane
}

\author{
L. Wang and F. J. Sigworth \\ Department of Cellular and Molecular Physiology, Yale University, New Haven, CT
}

Single-particle reconstruction (SPR) is an increasingly powerful method for determining the structure of proteins and other macromolecular assemblies without the need for a crystalline specimen [1]. Combining cryo-EM images from thousands of randomly-oriented macromolecular "particles", SPR allows three-dimensional structures to be obtained. For SPR structure determination of membrane proteins, traditionally the proteins are extracted from their membrane environment with detergent before rapid-freezing in vitreous ice and cryo-EM imaging. The detergent extraction renders proteins involved in membrane transport and signaling functions incapable of performing their normal function, and sometimes places them into non-native conformations. EM methods exist for obtaining the structures of membrane-incorporated proteins in special cases: two-dimensional crystalline arrays can sometimes be formed of proteins in a lipid membrane [2], and H. Saibil and colleagues [3] have demonstrated SPR of membrane protein "particles" imaged at the edge of large lipid vesicles. Our goal has been to develop a method that can be applied more generally.

The method, random spherically-constrained (RSC) single-particle reconstruction, allows SPR structure determination of membrane proteins reconstituted into small, spherical liposomes. We have employed this new method to obtain the structure of a $500 \mathrm{kDa}$ ion channel complex, the BK calcium-activated potassium channel, at $\sim 2 \mathrm{~nm}$ resolution [4]. From about 800 micrographs, 8000 protein particle images were selected and used in the reconstruction, as demonstrated in the figures.

The steps in the RSC-SPR procedure are the following.

1. The protein of interest is expressed in a cell line, extracted with detergent, and purified. Lipid is added and detergent is removed to reconstitute the protein into artificial lipid membranes, i.e. liposomes of $\sim 50 \mathrm{~nm}$ diameter. The protein-to-lipid ratio is chosen so that only one or a few copies of the protein are present in each liposome.

2. The liposomes are osmotically shocked and swollen to make them highly spherical, and are fast-frozen in vitreous ice of $\sim 100 \mathrm{~nm}$ thickness.

3. A quantitative model of the electron scattering of a lipid membrane is used to fit and subtract the membrane contribution to the image of each liposome. The diameter of liposomes varies greatly, so that the only way to do a 3D reconstruction is by "removing" the membrane contribution to the images in this way.

4. The residual "particle images" are used for single-particle reconstruction. The reconstruction process is greatly aided by the geometric constraints of the spherical liposome. In each image, the position of the particle relative to the center of the liposome provides information about two of the three Euler angles of orientation of the particle. The third angle and other ambiguities are resolved by a standard projection-matching procedure, and the determined angles for each particle image are used to compute a 3D density map by Fourier synthesis. 
5. If desired, the membrane density can be restored to the density map. Given the deduced angles of orientation of each particle and the membrane scattering model, the EM image of a small patch of membrane surrounding a particle is simulated and added back to the particle's image. A reconstruction from this set of images shows the protein particle in the membrane.

\section{References}

[1] J. Frank, Three Dimensional Electron Microscopy of Macromolecular Assemblies. (2006)

[2] S. Ranuserk and T. Walz, Ann. Rev. Biophys. 38 (2009) 89.

[3] S. J. Tilley et al. Cell 121 (2005) 247.

[4] L. Wang and F. J. Sigworth, Nature 461 (2009) 292.

[5] This work was supported by NIH grant NS 21501 to F. J. S.

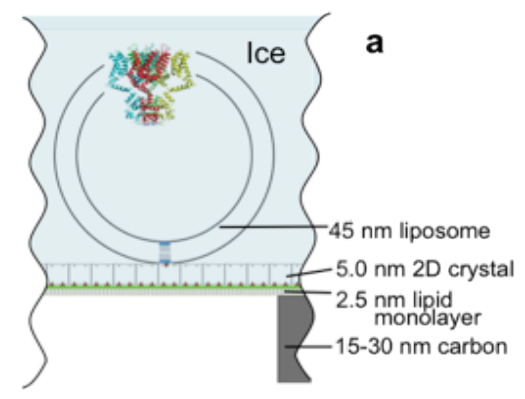

Figure 1. BK channel specimen and image processing. A, scale drawing of the specimen. A 2D streptavidin crystal spans holes in a perforated carbon film. Proteoliposomes doped with biotinylated lipid adhere to the crystal substrate. B, Portion of a CCD image taken with a Tecnai F30 microscope with GIF energy filer, at $300 \mathrm{keV}$ and $2.7 \mu \mathrm{m}$ defocus. C, Image after removal of the periodic crystal contribution. Inset shows an example liposome model with simulated protein particles. D, Image after membrane subtraction. Protein particles are boxed.
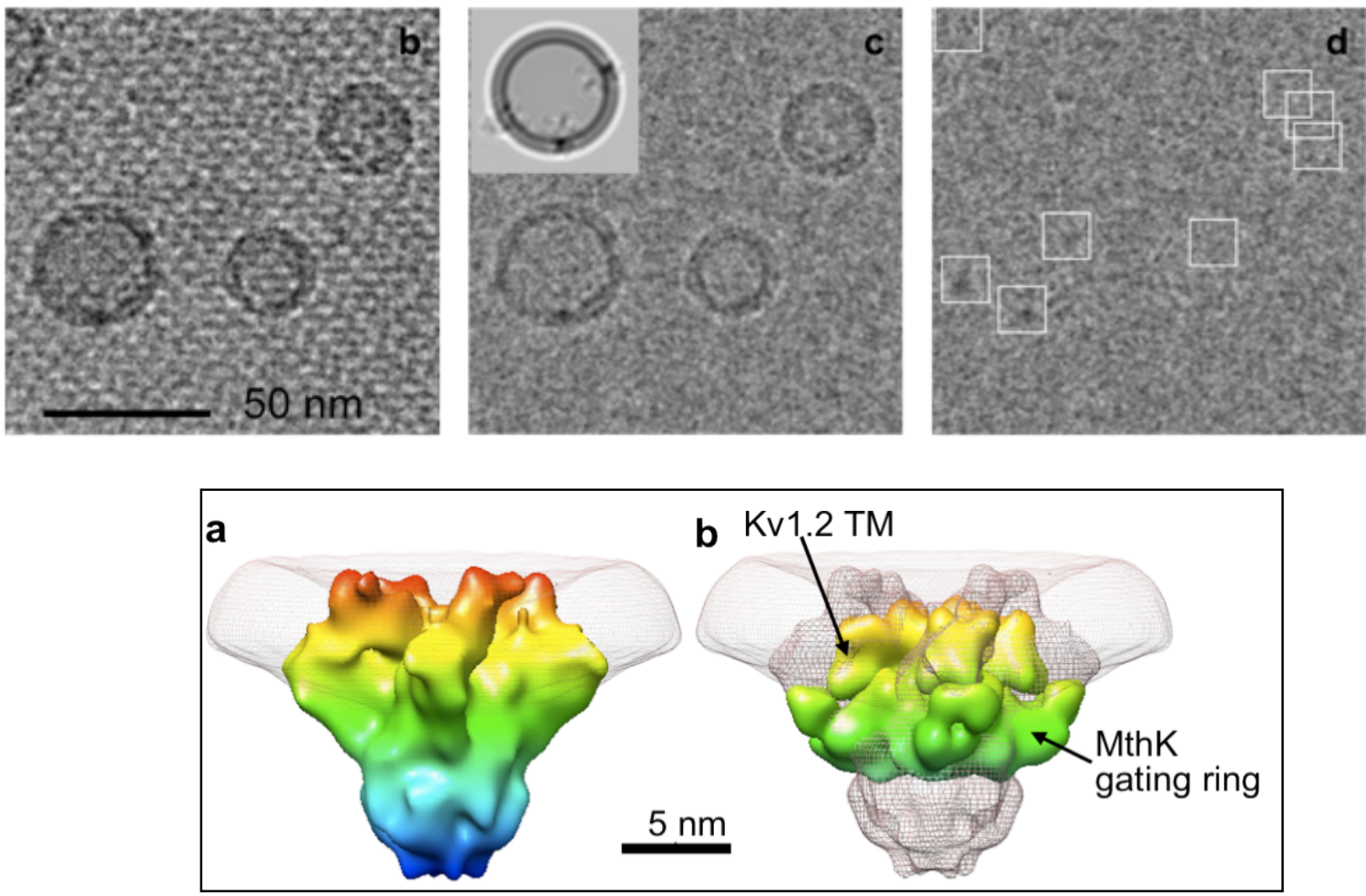

Figure 2. A, Rendering of the BK channel map. Restored membrane density is shown as light gray. The channel is oriented with its large cytoplasmic domain extending outward from the curved liposome membrane. B, Docking of the X-ray structures of homologous protein domains, filtered to $1.7 \mathrm{~nm}$ 